\title{
Severe falciparum malaria treated with artesunate complicated by delayed onset haemolysis and acute kidney injury
}

\author{
Katherine Plewes ${ }^{1,2,3^{*}}$, Md Shafiul Haider ${ }^{4}$, Hugh W F Kingston ${ }^{1,5}$, Tsin W Yeo ${ }^{5}$, Aniruddha Ghose ${ }^{4}$, \\ Md Amir Hossain ${ }^{4}$, Arjen M Dondorp ${ }^{1,2}$, Gareth D H Turner ${ }^{1,2}$ and Nicholas M Anstey ${ }^{5}$
}

\begin{abstract}
Background: Severe falciparum malaria may be complicated by haemolysis after parasite clearance, however the mechanisms remain unclear. Recent reports describe a pattern of delayed onset haemolysis among non-immune travellers with hyperparasitaemia treated with intravenous artesunate, termed post-artesunate delayed haemolysis (PADH). The occurrence and clinical impact of PADH following severe malaria infections in areas of unstable transmission are unknown.

Case: A 45-year-old Bangladeshi male was initially admitted to a local hospital with severe falciparum malaria complicated by hyperparasitaemia and treated with intravenous artesunate. Twenty days from his first presentation he was readmitted with delayed onset haemolytic anaemia and acute kidney injury. Multiple blood transfusions and haemodialysis were required. Renal biopsy revealed acute tubular injury and haem pigment nephropathy. His haemoglobin and renal function recovered to baseline after 62 days from his second admission.

Discussion: This case highlights the differential diagnosis of post-malaria delayed onset haemolysis, including the recently described syndrome of post-artemisinin delayed haemolysis. The pathophysiology contributing to acute kidney injury in this patient and the limited treatment options are discussed.

Conclusions: This report describes PADH complicated by acute kidney injury in an adult patient living in a malaria hypoendemic region who subsequently required blood transfusions and haemodialysis. This case emphasizes the importance of routine follow up of haemoglobin and renal function in artesunate-treated patients who have recovered from severe malaria.
\end{abstract}

Keywords: Falciparum malaria, Delayed haemolysis, Acute kidney injury

\section{Background}

Delayed onset haemolytic anaemia is a recognized but infrequent complication during recovery after severe falciparum malaria infection. The course of anaemia following severe malaria is often protracted and, in those treated with quinine, haemoglobin may not return to baseline until 28 days after infection [1]. Prior to the introduction of artesunate, prolonged haemolysis

\footnotetext{
*Correspondence: katherine@tropmedres.ac

${ }^{1}$ Mahidol Oxford Tropical Medicine Research Unit, Faculty of Tropical

Medicine, Mahidol University, Bangkok, Thailand

Full list of author information is available at the end of the article
}

contributed to this delayed haemoglobin recovery in less than $7 \%$ of patients [1]. The mechanisms of continued haemolysis after parasite clearance are unclear, with both auto-immune and non-immune processes implicated in the absence of artesunate treatment [2-4]. Recent reports describe a syndrome termed post-artemisinin delayed haemolysis (PADH) following treatment with intravenous artesunate [5-11]. PADH usually occurs 1-3 weeks after artesunate is administered to non-immune travellers with high parasitaemia. The different patterns of haemolysis following malaria infection and artesunate treatment highlight the complexity of malaria anaemia. 
Massive haemolysis is frequently associated with haemoglobinuria and acute kidney injury (AKI) [12-15]. When haemoglobin and haem scavengers, haptoglobin and haemopexin, are saturated the haemoglobin is filtered through the glomerulus. It is then re-absorbed and catabolized in the proximal tubule. Haemoglobinuria results when re-absorption capacity is reached [16]. Studies implicate cell-free haemoglobin in the mechanism of AKI in haemolytic conditions [17, 18]. This report describes the first case of a patient from an unstable malaria transmission area presenting with delayed-onset haemolysis complicated by acute kidney injury following treatment of severe malaria with intravenous artesunate and follow-on oral artemether lumefantrine combination treatment.

\section{Case presentation}

A 45-year-old, previously well, Bangladeshi male presented to Chittagong Medical College Hospital with a 4-day history of fever, headache, prostration, myalgias, nausea, and anorexia. This center is the main referral hospital for the Chittagong Division in southern Bangladesh, which is endemic for malaria with unstable transmission. One day prior he was diagnosed with malaria based on a positive rapid diagnostic test (RDT).

There was no significant past medical history, specifically no prior malaria infections, chronic illness, jaundice, liver or kidney disease, transfusions, malignancy, or autoimmune disease. There was no history of passing red or black urine. He was not taking any regular prescription or traditional medications and had no prior exposure to artemisinins.

On admission (day 0), his temperature was $40.3^{\circ} \mathrm{C}$, Glasgow Coma Score (GCS) 15/15, blood pressure (BP) 117/62 mmHg; heart rate (HR) 102 beats per minute (bpm) and respiratory rate 36 breaths per minute with $98 \%$ oxygen saturation. He appeared well with mild dehydration and scleral icterus, but otherwise unremarkable examination. He was diagnosed with uncomplicated malaria on admission and received one dose of oral artemether/lumefantrine combination (AL) $80 / 480 \mathrm{mg}$ (Novartis) since he had no clinical criteria for severe malaria, no initial blood tests and only an RDT-positive test.

Subsequent blood tests confirmed a diagnosis of severe falciparum malaria based on hyperparasitaemia (13.8\% erythrocytes infected; 659,000 parasites $/ \mu \mathrm{L}$ ) and hyperbilirubinaemia (bilirubin $80 \mu \mathrm{mol} / \mathrm{L}$ ). He was promptly switched to intravenous artesunate $120 \mathrm{mg}, 2.4 \mathrm{mg} /$ kg body weight, (Guilin No. 2, Pharmaceuticals, China) and received three doses at 12 -h intervals. He was also randomized to the normal saline arm of a randomized controlled trial of adjunctive L-arginine in severe malaria
(ACTRN12612000571875). After $24 \mathrm{~h}$ he defervesced and was switched to oral AL for 3 days (total of $480 \mathrm{mg}$ artemether and 2,880 $\mathrm{mg}$ lumefantrine). His recovery was unremarkable except for a delayed parasite clearance (estimated half life $8.2 \mathrm{~h}$ ). Due to this persistent parasitaemia, he received a second 3-day course of AL and his parasites cleared at $148 \mathrm{~h}$. In total he received 13 doses of $\mathrm{AL}$ (total dose of $1,040 \mathrm{mg}$ of artemether and $6,240 \mathrm{mg}$ of lumefantrine) and three doses of intravenous artesunate (total dose of $360 \mathrm{mg}$ ). He was discharged 8 days later with full recovery, a stable haematocrit and creatinine at a baseline of $86 \mu \mathrm{mol} / \mathrm{L}$.

At follow-up on day 14 after admission he was asymptomatic, however his white blood count revealed a significant leukocytosis and severe normocytic anaemia (haemoglobin of $60 \mathrm{~g} / \mathrm{L}$; Table 1). He declined re-admission and subsequently experienced fever, rigors, headache, jaundice, abdominal pain, and vomiting. He had not taken any antibiotics, non-steroidal anti-inflammatories or proton pump inhibitors during this intercurrent period after treatment of the initial malaria episode.

He was re-admitted on day 20 feeling unwell with generalized oedema, particularly of the face and lower extremities. Vital signs were stable: temperature $36.5^{\circ} \mathrm{C}$, GCS 15, BP 130/74 mmHg and HR $78 \mathrm{bpm}$. He appeared sick, anaemic and had periorbital oedema. Cardiac examination revealed a systolic flow murmur at the left upper sternal border with no radiation. There was mild abdominal tenderness on palpation and bilateral lower limb pitting oedema. Biochemistry revealed AKI with a creatinine increased to $1,769 \mu \mathrm{mol} / \mathrm{L}$, hyperkalaemia, a nonanion gap hyperchloraemic metabolic acidosis and severe anaemia with $4+$ haemoglobinuria (Table 1), with other investigations outlined below. Bladder catheterization did not suggest urinary retention with a residual volume of less than $50 \mathrm{~mL}$ of dark orange urine. He promptly received calcium gluconate and insulin for hyperkalaemia, empiric ceftriaxone, and was referred for haemodialysis and blood transfusion. Following five rounds of haemodialysis and three units whole blood transfusion, his metabolic acidosis, hyperkalaemia, renal function, and anaemia improved (Figure 1).

$\mathrm{He}$ was discharged recovering well on day 44 with stable serum creatinine and haemoglobin. He was followed up weekly thereafter, and by day 81 his kidney function improved further towards baseline (creatinine $115 \mu \mathrm{mol} / \mathrm{L})$, and his haemoglobin recovered $(135 \mathrm{~g} / \mathrm{L})$.

\section{Investigations}

Investigations are summarized in Table 1. Samples sent to Thailand and Australia for specialized tests are indicated by an asterisk. On day 20, reticulocytes accounted for $3 \%$ of erythrocytes (reticulocyte production index 
Table 1 Investigations

\begin{tabular}{|c|c|c|c|c|c|c|}
\hline Parameter & Day 0 & Day 3 & Day 14 & Day 20 & Day 44 & Normal range \\
\hline Plasma haemoglobin $(\mathrm{ng} / \mathrm{mL})^{a}$ & 177,839 & - & - & 127,146 & - & $<124,000$ \\
\hline Haemoglobin $(\mathrm{g} / \mathrm{L})$ & 141 & 136 & 60 & 49 & 102 & $140-180$ \\
\hline Haematocrit (\%) & 43.6 & 40 & 18.7 & 16 & 27.1 & $47-54$ \\
\hline Mean corpuscule volume (fL) & 86.3 & - & 86.2 & 94.7 & 85.2 & $76-96$ \\
\hline Plasma haptoglobin $(\mu \mathrm{g} / \mathrm{mL})^{a}$ & 1,160 & - & - & 5 & - & $300-2,000$ \\
\hline Platelets $\left(\times 10^{3} / \mu \mathrm{L}\right)$ & 28 & - & 228 & 478 & 320 & $150-00$ \\
\hline White blood cells $\left(\times 10^{3} / \mu \mathrm{L}\right)$ & 6.23 & - & 19.9 & 9.8 & 10.5 & $4-10$ \\
\hline Neutrophils (\%) & 83 & & - & 70 & 60 & $40-70$ \\
\hline Creatinine ( $\mu \mathrm{mol} / L)$ & 97 & 86 & - & 1,769 & 132 & $53-115$ \\
\hline Potassium ( $\mu \mathrm{mol} / \mathrm{L})$ & 3.7 & 4.4 & - & 8.4 & 4.8 & $3.5-4.9$ \\
\hline Venous pH & 7.41 & - & - & 7.2 & - & $7.31-7.41$ \\
\hline Venous bicarbonate (mmol/L) & 19.5 & - & - & 8.7 & - & $23-28$ \\
\hline Venous carbon dioxide (mmHg) & 31.2 & - & - & 23.5 & - & $41-51$ \\
\hline Venous base excess (mmol/L) & -5 & - & - & -20 & - & $(-2)$ to $(+3)$ \\
\hline Venous lactate (mmol/L) & 2.36 & - & - & 0.58 & - & $0.9-1.7$ \\
\hline Anion gap (mmol/L) & 13 & - & - & 6 & - & $10-20$ \\
\hline Total bilirubin ( $\mu \mathrm{mol} / \mathrm{L})$ & 80 & - & - & - & - & $3-21$ \\
\hline Indirect bilirubin ( $\mu \mathrm{mol} / \mathrm{L})$ & 26 & - & - & - & - & $2-14$ \\
\hline Lactate dehydrogenase (U/L) & 592 & - & - & 887 & - & $140-280$ \\
\hline International normalization ratio & - & 1.3 & - & - & - & $0.8-1.2$ \\
\hline Partial thromboplastin time (seconds) & - & 15 & - & - & - & $<30$ \\
\hline
\end{tabular}

a Specialized tests performed on samples sent to Darwin, Australia.

0.4). The direct antiglobulin test (DAT; anti-IgG and antiC3d) and indirect antiglobulin test (IAT) were negative. Plasma cell-free haemoglobin* was elevated (127,000 ng/ $\mathrm{mL}$; normal range $<124,000 \mathrm{ng} / \mathrm{mL}$ ), and haptoglobin* decreased (5 $\mu \mathrm{g} / \mathrm{mL}$; normal range: $300-2,000 \mu \mathrm{g} / \mathrm{mL}$ ). A 24-h urine collection revealed $525 \mathrm{mg}$ of protein (normal range $<150 \mathrm{mg} /$ day). Urine microscopy showed red blood cells casts and dysmorphic red cells. Urine biochemistry*: osmolarity $283 \mathrm{mOsm} / \mathrm{kg}$, sodium $84 \mathrm{mmol} / \mathrm{L}$, urine anion gap of 8 , urine osmolar gap 21, and transtubular potassium gradient 1.8. Anti-nuclear antibodies were absent and $\mathrm{C} 3 / \mathrm{C} 4$ levels were normal. HIV and hepatitis $\mathrm{C}$ antibodies, hepatitis $\mathrm{B}$ surface and envelope antigen were negative. Blood cultures and bacterial $16 \mathrm{~S}^{*}$ ribosomal DNA amplification performed to exclude bacteraemia as a cause of the leukocytosis were negative. Repeat peripheral blood smears for malaria were negative and no schistocytes were found. Glucose-6-phosphate dehydrogenase (G6PD) was qualitatively normal on day 0 .

Abdominal ultrasound on day 22 showed normal kidney size with increased parenchymal echogenicity and poor corticomedullary differentiation, with no pelvicalyceal system dilatation.

A renal biopsy performed on day 27 revealed severe and recovering acute tubular injury, with interstitial oedema but only minimal tubulointerstitial inflammation
(Figure 2). Tubules showed acute tubular dilatation, injury and regenerative features. Several red cell casts and amorphous casts were present. Vessels were normal. Glomeruli were morphologically normal with no active glomerulonephritis, negative immunoperoxidase staining for immunoglobulins and C3, and no immune complex deposition or basement membrane abnormalities on electron microscopy. A myoglobin immunostain was negative in casts. However, a Perl's stain for iron showed both diffuse tubular staining and increased deposition in granular deposits within the cytoplasm of damaged tubular epithelial cells.

\section{Discussion}

This report describes a case of post-artesunate delayedonset haemolysis complicated by acute kidney injury after recovery from severe falciparum malaria with hyperparasitaemia. Identifying the cause of haemolytic anaemia following malaria infection is challenging, as its mechanism is multifactorial and the broad differential diagnosis requires specialized testing that is often unavailable in resource-limited settings. The timing of haemolysis in this case 14 days after the first dose of artesunate accompanied by a $>10 \%$ drop in haemoglobin (from day 7 haemoglobin), haptoglobin $<0.1 \mathrm{~g} / \mathrm{L}$ and lactate dehydrogenase $>390 \mathrm{U} / \mathrm{L}$ is consistent with 

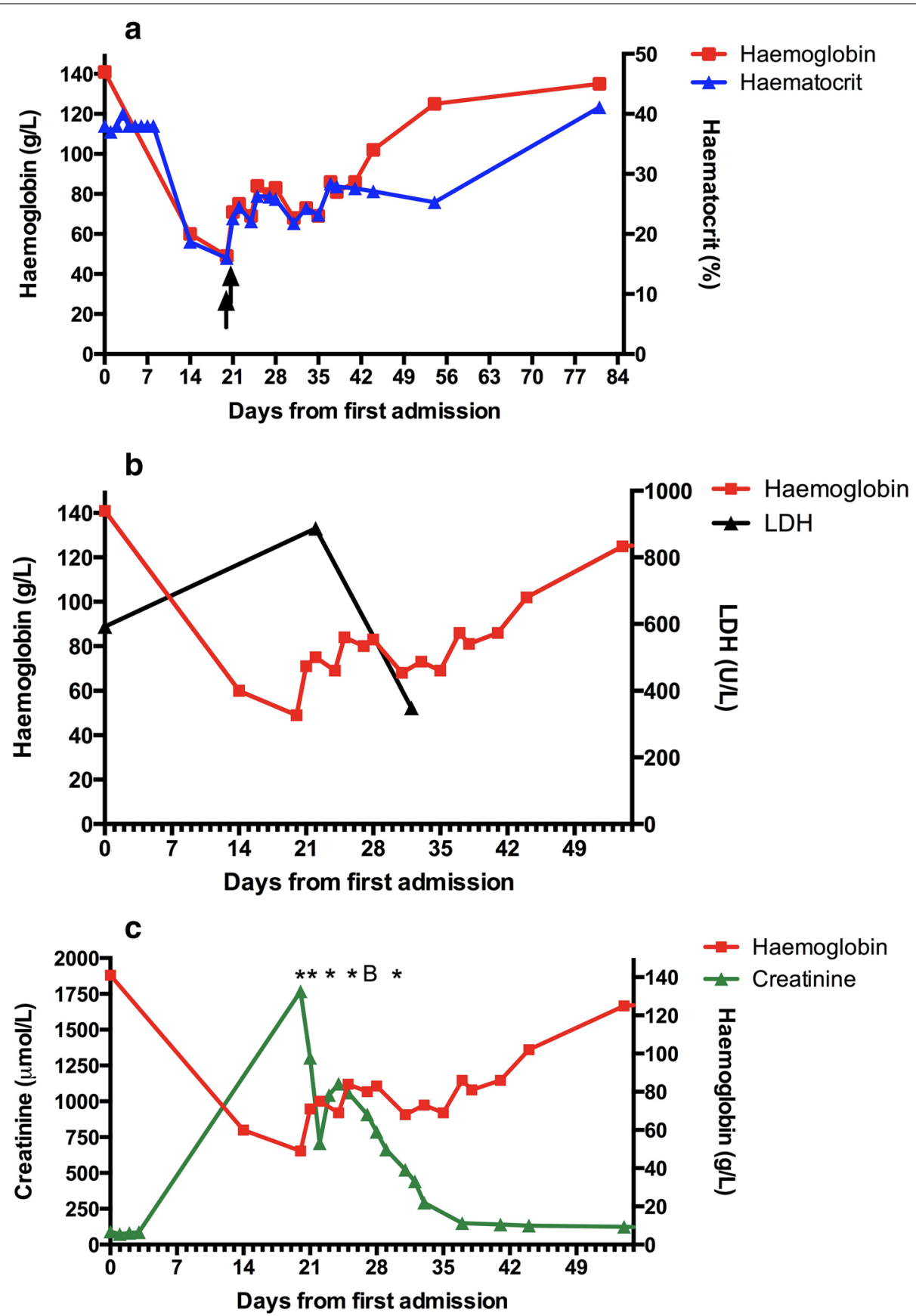

Figure 1 Haemoglobin, haematocrit, creatinine, and lactate dehydrogenase profiles from both hospital admissions. The first admission for severe malaria with hyperparasitaemia and hyperbilirubinaemia from day 0 to day 8; second hospital admission for severe haemolytic anaemia and acute kidney injury from day 20 to day 44. a Haemoglobin and haematocrit profile during hospital admissions. Haematocrit was stable during first hospital admission. Haemoglobin dropped by 57 and $65 \%$ on days 14 and 20, respectively, and recovered by day 81 . Arrows indicate timing of whole blood transfusions on days 20 and 21; b haemoglobin and lactate dehydrogenase (LDH) profile during hospital admissions; $\mathbf{c}$ haemoglobin and creatinine profile during hospital admissions. Acute kidney injury, with a creatinine increase by $21 \times$ on second hospital admission, classified as stage 3 according to AKIN criteria and 'failure' by RIFLE criteria. Presumed baseline creatinine from day 1 was $74 \mu$ mol/L. His creatinine returned close to baseline by day 81 (106 $\mathrm{mmol} / \mathrm{L}$ ). Asterisks indicate haemodialysis on days 20,21, 23, 25, and 30;' $B^{\prime}$ indicates renal biopsy on day 27 . AKIN acute kidney injury network, RIFLE risk, injury, failure, loss, end-stage kidney disease. 

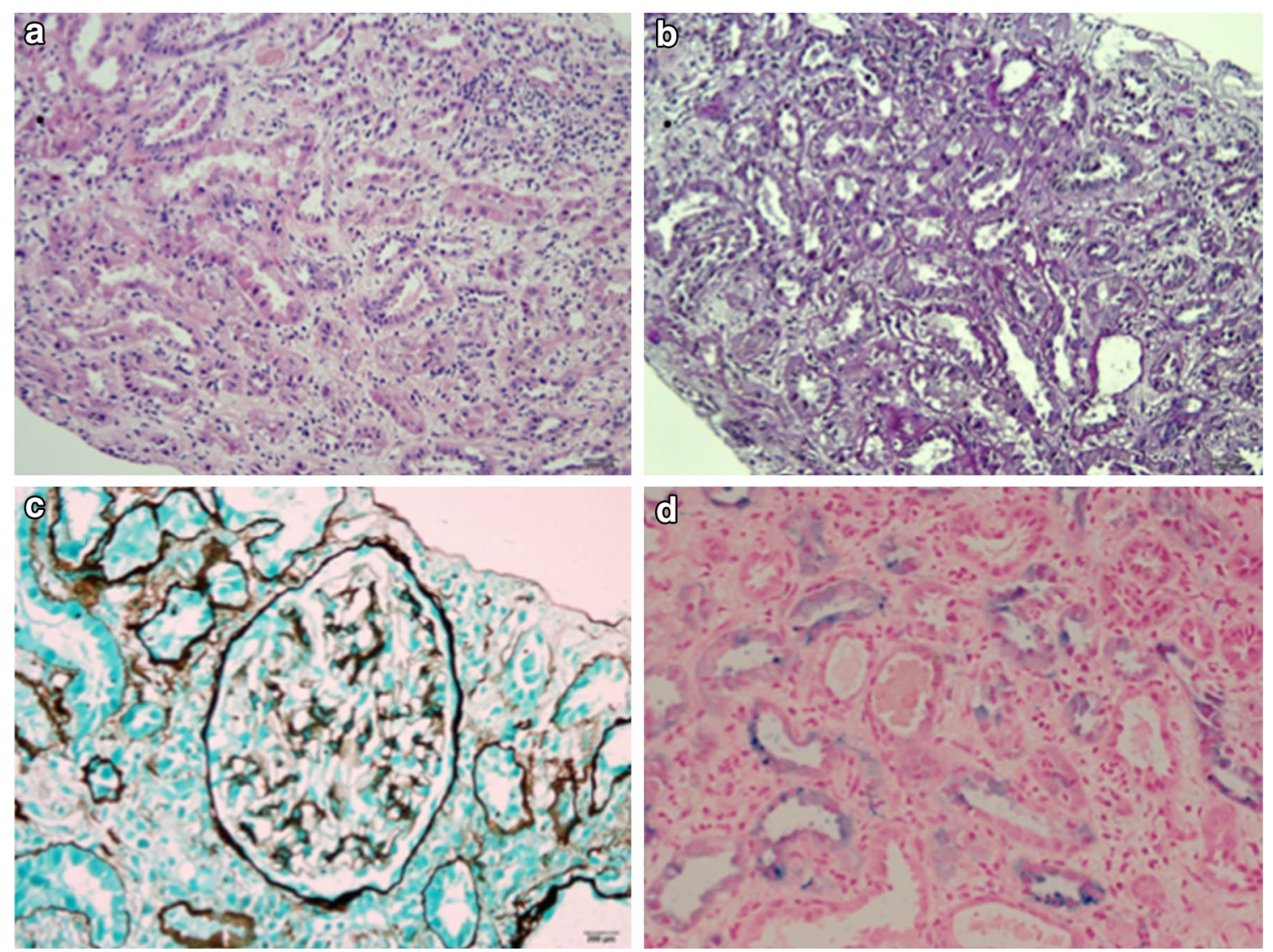

Figure 2 Renal biopsy. Left kidney ultrasound guided biopsy performed on day 27. Photomicrographs to show histopathological findings; a acute tubular necrosis with dilation, epithelial thinning, tubular cell necrosis and regeneration, interstitial oedema, and minimal inflammation (haematoxylin and eosin stain $\times 200$ ); $\mathbf{b}$ acute tubular injury with PAS staining showing loss of brush border in the dilated injured proximal tubules (Periodic acid Schiff $\times 200$ ); $\mathbf{c}$ a normal glomerulus ( $\times 400$ Jones methanamine silver stain), and $\mathbf{d}$ a Perls stain for iron showing particulate and diffuse staining in damaged proximal tubules ( $\times 400)$. Specialized PAS, methanamine, and Perls straining were performed in Bangkok, Thailand since only haematoxylin and eosin stain was available in Bangladesh.

the case definition of PADH $[5,7,8]$. Since 2012, PADH is proposed to have occurred in at least 38 patients [19, 20], however these reports do not strictly adhere to the optimized definition of PADH recently published in 2014 [5, 7]. Given this limitation it is difficult to accurately determine the frequency and clinical impacts of PADH. A recent review of the reported cases with an alternative PADH case definition estimates that among severe malaria patients treated with intravenous artesunate PADH occurs in 13\% and blood transfusions are required in 9\% [20]. Of these PADH cases, 31 were non-immune returning travellers, six were immune African children under 5 years, and one was an immune West African adult [6, 9-11, 20-26]. The case reported here describes PADH in a non-immune adult Bangladeshi patient who recovered from an episode of severe malaria acquired while living in an unstable malaria transmission region.
The mechanism of PADH is hypothesized to be due to pitting of ring staged parasitized red cells, where the spleen removes the dead parasite from the red cell akin to the removal of Howell-Jolly bodies [5]. These pitted cells are formed quickly after treatment with artesunate and have a shortened red cell survival [27-29]. Destruction of the once-infected pitted cells manifests as delayed onset of haemolysis. However, in this case and other reported cases of PADH [30] the magnitude of the drop in haemoglobin exceeds the fall expected if only the once-infected pitted cells are destroyed. This suggests that additional artesunate-associated mechanisms of delayed haemolysis involving uninfected red cells must occur. Inhibition of erythropoiesis has also been proposed to contribute to the anaemia as it has been suggested that patients receiving higher doses of artesunate are at higher risk of bone marrow suppression [7]. This may have contributed to the anaemia in the presented case as he received a total 
dose of $7.2 \mathrm{mg} / \mathrm{kg}$ artesunate and $20.8 \mathrm{mg} / \mathrm{kg}$ artemether, and a day 20 reticulocyte production index of $<2 \%$ supported an inadequate bone marrow response to his haemolysis. The young ring-stage activity of artesunate is one characteristic that confers its life-saving benefit over quinine. Artesunate remains first-line anti-malarial for the treatment of severe malaria since the benefit of survival outweighs the low probability of non-life-threatening delayed haemolysis. Recent guidelines recommend weekly monitoring malaria patients for indicators of haemolysis for 1 month following treatment with artesunate [30].

This case of delayed haemolysis was complicated by severe AKI, which has not been reported in the setting of PADH. The presence of excess haem pigment in tubular cells may be due to re-absorption from tubular red cells or haemoglobinuria. The role of haemoglobinuria has been associated with AKI in a spectrum of diseases including blackwater fever, babesiosis, paroxysmal nocturnal haemoglobinuria, massive transfusion and post-cardiopulmonary bypass $[12-15,17,18,31]$. While not all cases of haemoglobinuria result in AKI, up to $64 \%$ of patients with blackwater fever develop renal dysfunction [14]. In this case the features suggested direct tubular epithelial toxicity due to free haem rather than a cast nephropathy. The pathophysiology of haem-pigment AKI is not completely defined but vasoconstriction; direct tubular cell injury and tubular obstruction are thought to contribute [32]. There is accumulating evidence that haemoproteins induce oxidative renal damage by lipid peroxidation [17, 33]. The redox cycling of the haem group initiates lipid peroxidation, releasing potent oxidative compounds that cause renal vasoconstriction [33]. This mechanism is demonstrated in post-operative AKI after post-cardiopulmonary bypass where high cell-free haemoglobin and oxidative stress were associated with kidney injury [17, 18]. Cell-free haemoglobin is also likely to cause vasoconstriction through its quenching of endothelial nitric oxide (NO) in severe falciparum malaria, where it has been associated with impairment in NO-dependent endothelial function [34]. The renal pathology in this case is consistent with haem-pigment induced AKI with clinical evidence of non-anion gap hyperchloraemic metabolic acidosis with distal tubule dysfunction likely secondary to hypovolaemia and vasoconstriction.

The differential diagnosis for haemolysis in this case is broad and the possible contribution of an intercurrent bacterial infection between admissions was considered given his leukocytosis on day 14. Infections may cause haemolysis by precipitating oxidative stress in patients with underlying red blood cell (RBC) disorders, inducing haemolytic pathologic responses, or from the antimicrobial therapy itself [35]. Limited diagnostics suggested no underlying RBC abnormality: normal qualitative G6PD, mean corpuscular volume (MCV) and peripheral smear. Negative DAT and IAT argued against an immune-mediated haemolytic anaemia. Infections characterized by haemolysis include: malaria, babesiosis, Carrion's disease, and typhoid. The peripheral smear on second admission revealed no intra-erythrocytic organisms. Negative blood cultures and bacterial 16S ribosomal DNA amplification were not conclusive but argued against infection as a cause of the haemolysis. Furthermore, the leukocytosis observed on day 14 had resolved by day 20 in the absence of antibiotic treatment.

The differential diagnosis for the etiology of AKI included (1) pre-renal, and (2) intrinsic renal causes including: acute tubular injury (ATI) due to ischaemia or direct toxin-mediated injury by cell-free haemoglobin, acute interstitial nephritis (AIN) secondary to $\beta$-lactams, microangiopathy, or post-infectious glomerulonephritis (PIGN). The urinary osmolarity $<350 \mathrm{mOsm} / \mathrm{kg}$ and fractional excretion of sodium $>1 \%$ did not suggest a pre-renal cause. The absence of systemic eosinophils and eosinophil-rich tubulo-interstitial inflammation on renal biopsy argued against a diagnosis of AIN, although recovering AIN can be pauci-inflammatory. The normal platelets, lack of schistocytes, negative blood cultures and 16S rRNA amplification, and normal renal vessels and glomeruli on biopsy were not consistent with haemolytic uraemia syndrome/thrombotic thrombocytopenic purpura or PIGN.

Treatment for established AKI is limited to renal replacement therapy. Research towards prevention and alternative treatments is vital since dialysis is frequently unavailable. Currently, there is no targeted treatment for cell-free haemoglobin-induced AKI, however results from experimental models and case series are encouraging. Recent studies have shown that paracetamol inhibits haemoprotein-induced lipid peroxidation, resulting in decreased oxidative kidney injury and improved renal function [36, 37]. Administration of haptoglobin infusions is shown to attenuate cell-free haemoglobin-mediated AKI in paroxysmal nocturnal haemoglobinuria and an experimental model of stored blood transfusion [38, 39].

\section{Conclusions}

Malaria treatment guidelines should consider recommending that renal function be checked if a drop in haemoglobin is detected during monitoring of severe malaria patients after treatment with artesunate. This may be logistically difficult in limited-resource countries but where it can be done, monitoring the creatinine should be a part of severe malaria management to detect and avoid renal complications. 


\section{Learning points}

Delayed onset haemolytic anaemia following parasite clearance in severe malaria infection has a broad differential diagnosis to consider.

Delayed onset haemolysis following severe malaria may occur following artesunate treatment, and be complicated by AKI.

Severe malaria patients treated with artesunate should be monitored weekly for decline of haemoglobin and renal function.

There are limited treatment options to treat haemoprotein-mediated AKI.

\begin{abstract}
Abbreviations
AIN: acute interstitial nephritis; AKl: acute kidney injury; AL: artemether lumefantrine; ATI: acute tubular injury; BP: blood pressure; bpm: beats per minute; DAT: direct antiglobulin test; G6PD: glucose-6-phosphate dehydrogenase; GCS: Glasgow coma score; HIV: human immunodeficiency virus; HR: heart rate; IAT: indirect antiglobulin test; MCV: mean corpuscular volume; NO: nitric oxide; PADH: post-artesunate delayed haemolysis; PIGN: post-infectious glomerulonephritis; RBC: red blood cell; RDT: rapid diagnostic test.
\end{abstract}

\section{Authors' contributions}

All authors contributed to the content of this case report. KP performed the clinical assessments, data collection and drafted manuscript and figures. MSH performed clinical assessments, guided dialysis and performed renal biopsy. $H K, T Y$ and NMA performed clinical assessments. AG and AH were responsible for clinical care of the patient. GDHT reviewed the renal biopsy, and assisted in drafting the manuscript and figures. HK, TWY, AMD, GDHT, and NMA critically interpreted the clinical case, and revised manuscript for intellectual content. All authors read and approved the final manuscript.

\section{Author details}

${ }^{1}$ Mahidol Oxford Tropical Medicine Research Unit, Faculty of Tropical Medicine, Mahidol University, Bangkok, Thailand. ${ }^{2}$ Nuffield Department of Medicine, Centre for Tropical Medicine and Global Health, University of Oxford, Oxford, UK. ${ }^{3}$ Division of Infectious Diseases, Department of Medicine, University of British Columbia Clinical Investigator Program, Vancouver, Canada. ${ }^{4}$ Department of Medicine, Chittagong Medical College Hospital, Chittagong, Bangladesh. ${ }^{5}$ Global Health Division, Menzies School of Health Research and Charles Darwin University, Darwin, NT, Australia.

\section{Acknowledgements}

We thank the patient, medical residents, attending physicians, and support staff at Chittagong Medical College Hospital for their assistance and collaboration with the Mahidol-Oxford Tropical Medicine Research Unit, Kim Piera for laboratory assistance, and Dr. Claire Ling for molecular analysis. This work was supported by the Australian National Health and Medical Research Council (grant number 605807 and Fellowships to NMA and TWY). Mahidol-Oxford Tropical Medicine Research Unit is funded by the Wellcome Trust of Great Britain through grant number 089275/Z/09/Z. KP was supported by the University of British Columbia Clinician Investigator Program, Vancouver, Canada.

\section{Compliance with ethical guidelines}

\section{Competing interests}

The authors declare that they have no competing interests.

\section{Consent for publication}

Ethical approval was obtained from the Ministry of Health in Bangladesh, Menzies Human Research Ethics Committee in Australia, and the Oxford Tropical Research Ethics Committee, Oxford, UK. Written informed consent was obtained from the patient for publication of this case report and any accompanying images. A copy of the written consent is available for review by the Editor-in-Chief of Malaria Journal.
Received: 13 April 2015 Accepted: 2 June 2015

Published online: 18 June 2015

\section{References}

1. Camacho LH, Gordeuk VR, Wilairatana P, Pootrakul P, Brittenham GM, Looareesuwan S (1998) The course of anaemia after the treatment of acute, falciparum malaria. Ann Trop Med Parasitol 92:525-537

2. Adner MM, Altstatt LB, Conrad ME (1968) Coombs'-positive hemolytic disease in malaria. Ann Intern Med 68:33-38

3. Omodeo-Sale F, Motti A, Dondorp A, White NJ, Taramelli D (2005) Destabilisation and subsequent lysis of human erythrocytes induced by Plasmodium falciparum haem products. Eur J Haematol 74:324-332

4. Woodruff AW, Ansdell VE, Pettitt LE (1979) Cause of anaemia in malaria. Lancet 1:1055-1057

5. Jaureguiberry S, Ndour PA, Roussel C, Ader F, Safeukui I, Nguyen M et al (2014) Postartesunate delayed hemolysis is a predictable event related to the lifesaving effect of artemisinins. Blood 124:167-175

6. Kreeftmeijer-Vegter AR, van Genderen PJ, Visser LG, Bierman WF, Clerinx J, van Veldhuizen CK et al (2012) Treatment outcome of intravenous artesunate in patients with severe malaria in the Netherlands and Belgium. Malar J 11:102

7. Experts Group Meeting on delayed anaemia following treatment with injectable artesunate, Wien Austria. (http://www.mmv.org/sites/default/ files/uploads/docs/events/2013/InjectableArtesunateExpertGroupMeeting.pdf)

8. Paczkowski MM, Landman KL, Arguin PM (2014) Update on cases of delayed hemolysis after parenteral artesunate therapy for malariaUnited States, 2008 and 2013. MMWR Morb Mortal Wkly Rep 63:753-755

9. Rolling T, Agbenyega T, Issifou S, Adegnika AA, Sylverken J, Spahlinger D et al (2014) Delayed hemolysis after treatment with parenteral artesunate in African children with severe malaria-a double-center prospective study. J Infect Dis 209:1921-1928

10. Rolling T, Wichmann D, Schmiedel S, Burchard GD, Kluge S, Cramer JP (2013) Artesunate versus quinine in the treatment of severe imported malaria: comparative analysis of adverse events focussing on delayed haemolysis. Malar J 12:241

11. Zoller T, Junghanss T, Kapaun A, Gjorup I, Richter J, Hugo-Persson M et al (2011) Intravenous artesunate for severe malaria in travelers, Europe. Emerg Infect Dis 17:771-777

12. Blum S, Gattringer R, Haschke E, Walochnik J, Tschurtschenthaler G, Lang F et al (2011) The case: hemolysis and acute renal failure. Babesiosis. Kidney Int 80:681-683

13. Clark DA, Butler SA, Braren V, Hartmann RC, Jenkins DE Jr (1981) The kidneys in paroxysmal nocturnal hemoglobinuria. Blood 57:83-89

14. Tran TH, Day NP, Ly VC, Nguyen TH, Pham PL, Nguyen HP et al (1996) Blackwater fever in southern Vietnam: a prospective descriptive study of 50 cases. Clin Infect Dis 23:1274-1281

15. Tsai CW, Wu VC, Lin WC, Huang JW, Wu MS (2007) Acute renal failure in a patient with paroxysmal nocturnal hemoglobinuria. Kidney Int 71:1187

16. Bunn HF, Jandl JH (1969) The renal handling of hemoglobin. II. Catabolism. J Exp Med 129:925-934

17. Billings FT, Ball SK, Roberts $\sqcup$, Pretorius M (2011) Postoperative acute kidney injury is associated with hemoglobinemia and an enhanced oxidative stress response. Free Radic Biol Med 50:1480-1487

18. Vermeulen Windsant IC, Snoeijs MG, Hanssen SJ, Altintas S, Heijmans JH, Koeppel TA et al (2010) Hemolysis is associated with acute kidney injury during major aortic surgery. Kidney Int 77:913-920

19. Raffray L, Receveur MC, Beguet M, Lauroua P, Pistone T, Malvy D (2014) Severe delayed autoimmune haemolytic anaemia following artesunate administration in severe malaria: a case report. Malar J 13:398

20. Rehman K, Lotsch F, Kremsner PG, Ramharter M (2014) Haemolysis associated with the treatment of malaria with artemisinin derivatives: a systematic review of current evidence. Int J Infect Dis 29:268-273

21. Caramello P, Balbiano R, De Blasi T, Chiriotto M, Deagostini M, Calleri G (2012) Severe malaria, artesunate and haemolysis. J Antimicrob Chemother 67:2053-2054 
22. Corpolongo A, De Nardo P, Ghirga P, Gentilotti E, Bellagamba R, Tommasi C et al (2012) Haemolytic anaemia in an HIV-infected patient with severe falciparum malaria after treatment with oral artemether-lumefantrine. Malar J 11:91

23. De Nardo P, Oliva A, Giancola ML, Ghirga P, Mencarini P, Bibas M et al (2013) Haemolytic anaemia after oral artemether-lumefantrine treatment in a patient affected by severe imported falciparum malaria. Infection 41:863-865

24. Eder M, Farne H, Cargill T, Abbara A, Davidson RN (2012) Intravenous artesunate versus intravenous quinine in the treatment of severe falciparum malaria: a retrospective evaluation from a UK centre. Pathog Glob Health 106:181-187

25. Jarvis JN, Coltart CE, Pule M, Chiodini PL, Doherty T (2013) Artemisinin therapy and severe delayed haemolysis. Lancet 382:180

26. Kano S (2010) Artemisinin-based combination therapies and their introduction in Japan. J Infect Chemother 16:375-382

27. Angus BJ, Chotivanich K, Udomsangpetch R, White NJ (1997) In vivo removal of malaria parasites from red blood cells without their destruction in acute falciparum malaria. Blood 90:2037-2040

28. Chotivanich K, Udomsangpetch R, Dondorp A, Williams T, Angus B, Simpson JA et al (2000) The mechanisms of parasite clearance after antimalarial treatment of Plasmodium falciparum malaria. J Infect Dis 182:629-633

29. Newton PN, Chotivanich K, Chierakul W, Ruangveerayuth R, Teerapong P, Silamut K et al (2001) A comparison of the in vivo kinetics of Plasmodium falciparum ring-infected erythrocyte surface antigen-positive and -negative erythrocytes. Blood 98:450-457

30. Centers for Disease Control and Prevention (CDC) (2013) Published reports of delayed hemolytic anemia after treatment with artesunate for severe malaria-worldwide, 2010-2012. MMWR Morb Mortal Wkly Rep $62: 5-8$
31. Rubin H (1971) Paroxysmal nocturnal hemoglobinuria with renal failure. JAMA 215:433-436

32. Heyman SN, Rosen S, Fuchs S, Epstein FH, Brezis M (1996) Myoglobinuric acute renal failure in the rat: a role for medullary hypoperfusion, hypoxia and tubular obstruction. J Am Soc Nephrol 7:1066-1074

33. Moore KP, Holt SG, Patel RP, Svistunenko DA, Zackert W, Goodier D et al (1998) A causative role for redox cycling of myoglobin and its inhibition by alkalinization in the pathogenesis and treatment of rhabdomyolysisinduced renal failure. J Biol Chem 273:31731-31737

34. Yeo TW, Lampah DA, Tjitra E, Gitawati R, Kenangalem E, Piera Ket al (2009) Relationship of cell-free hemoglobin to impaired endothelial nitric oxide bioavailability and perfusion in severe falciparum malaria. J Infect Dis 200:1522-1529

35. Berkowitz FE (1991) Hemolysis and infection: categories and mechanisms of their interrelationship. Rev Infect Dis 13:1151-1162

36. Boutaud O, Moore KP, Reeder BJ, Harry D, Howie AJ, Wang S et al (2010) Acetaminophen inhibits hemoprotein-catalyzed lipid peroxidation and attenuates rhabdomyolysis-induced renal failure. Proc Natl Acad Sci USA 107:2699-2704

37. Janz DR, Bastarache JA, Peterson JF, Sills G, Wickersham N, May AK et al (2013) Association between cell-free hemoglobin, acetaminophen, and mortality in patients with sepsis: an observational study. Crit Care Med 41:784-790

38. Baek JH, D'Agnillo F, Vallelian F, Pereira CP, Williams MC, Jia Y et al (2012) Hemoglobin-driven pathophysiology is an in vivo consequence of the red blood cell storage lesion that can be attenuated in guinea pigs by haptoglobin therapy. J Clin Invest 122:1444-1458

39. Shibasaki T, Matsuda H, Furuya K (2007) Haptoglobin therapy during pregnancy for paroxysmal nocturnal hemoglobinuria with renal failure. Int J Gynaecol Obstet 98:267-268

\section{Submit your next manuscript to BioMed Central and take full advantage of:}

- Convenient online submission

- Thorough peer review

- No space constraints or color figure charges

- Immediate publication on acceptance

- Inclusion in PubMed, CAS, Scopus and Google Scholar

- Research which is freely available for redistribution

Submit your manuscript at

www.biomedcentral.com/submit

C Biomed Central 